\title{
Análisis físico, químico y detección de salmonella spp en huevos provenientes de gallus gallus domesticus, comunidad Piedra Larga, Condega 2018-2019
}

\section{Physical, chemical analysis of salmonella spp on eggs coming from gallus gallus domesticus, piedra larga, Condega community 2018-2019}

\author{
Medardo de Jesús Moreno Castellón', Esther Jalieth Velasquez Raudez ${ }^{2}$, Michelle Alexandra Moreno Rivera² \\ 1 Docente investigador de la facultad de Ciencias Agropecuarias -,UCATSE - Estelí Nicaragua \\ drmevet@hotmail.com \\ 2 Médico veterinario egresado UCATSE- Estelí Nicaragua \\ esterraudez@gmail.com; morenomr1007@gmail.com
}

Recibido: 15/feb/2021 Revisado: 30/abr/2021

Aceptado: 30/may/2021 Publicado: 30/jul/2021

Resumen El estudio se realizó en la comunidad Piedra Larga, municipio de Condega departamento de Estelí, en el cual tuvo como objetivo determinar los parámetros físicos, químicos y detección de Salmonella spp en cáscaras de huevos de gallinas de traspatio, mediante un estudio descriptivo de corte transversal, cuyo fin fue determinar la calidad de los huevos. Este se basó en el análisis de 95 huevos recolectados en 19 casas de la comunidad. Las variables evaluadas incluyeron grado de frescura mediante unidades Hauhg, gravedad especifica según la posición del huevo en grados, diámetro de clara, índice de yema, $\mathrm{pH}$ de clara y yema, altura de la cámara de aire, así mismo la determinación de color de yema, peso total del huevo y análisis de Salmonella spp en cáscara. Respecto a los resultados, se describe que las variaciones más relevantes en la calidad del huevo fueron en el análisis de grado de frescura con $50 \mathrm{UH}$, el incremento de $\mathrm{pH}$ en clara y en el peso total del huevo que fue menor de $53 \mathrm{gr}$, indicando que son de menor tamaño. En cuanto a la detección de Salmonella $s p p$, no se obtuvieron resultados significativos, ya que solamente se evidenció en una de las muestras. Con respecto al tipo de almacenamiento y al manejo que los pequeños productores les realizan a los huevos, se determinó que puede influir en la calidad e inocuidad interna del huevo, por lo tanto se determinó que los huevos son de baja calidad.

Palabras claves huevos; unidades Hauhg; Salmonella spp; altura; $\mathrm{pH}$.

\begin{abstract}
Abstrac The study was carried out in the Piedra Larga community, municipality of Condega, Estelí department, in which the objective of the study was to determine the physical and chemical parameters and detection of Salmonella spp in shell eggs of backyard chickens, through a descriptive cross-sectional study. whose purpose was to determine the quality of the eggs. This was based on the analysis of 95 eggs collected from 19 houses in the community. The variables evaluated included degree of freshness by Hauhg units, specific gravity according to the position of the egg in degrees, diameter of white, yolk index, $\mathrm{pH}$ of white and yolk, height of the air chamber, as well as the determination of yolk color. , total egg weight and analysis of Salmonella spp in shell. Regarding the results, it is described that the most relevant variations in the quality of the egg were in the analysis of the degree of freshness with $50 \mathrm{HU}$, the increase in $\mathrm{pH}$ in white and in the total weight of the egg, which was less than $53 \mathrm{gr}$, indicating that they are smaller. Regarding the detection of Salmonella spp, no significant, results were obtained since it was only evidenced in one of the samples. Regarding the type of storage and the handling that small producers carry out to the eggs, it was determined that it can influence the quality and internal safety of the egg, therefore it was determined that the eggs are of low quality
\end{abstract}

Keywords. eggs; Haugh units; Sallmonella spp, height; pH.

Teknos Revista Científica. | Volumen 21 No.1 - diciembre 2021 | ISSN 1900-7388 (papel) | ISSN 2539-2190 (digital)

DOI: https://doi.org/10.25044/issn.2539-2190 


\section{Introducción}

El consumo del huevo de gallina de patio por parte de la población, tiene mucha importancia por ser de gran valor nutritivo en la alimentación diaria del ser humano, pero debido a su compleja constitución, pueden sufrir alteraciones asociadas a cambios físicos, químicos y microbiológicos en sus constituyentes, especialmente como consecuencia de la genética, la edad de la gallina, el tiempo transcurrido luego de la ovoposición, hasta su comercialización y consumo, además del manejo y de las condiciones de almacenamiento (Rosero, 2015). Estas modificaciones son bien conocidas y refieren principalmente en el aumento del volumen de la cámara de aire, debilitamiento de la membrana vitelina y licuefacción de la parte densa de la albumina. (Estrada, Galeano, \& Her, 2010)

En Nicaragua la producción de gallinas de patio aporta el $5 \%$ de la producción nacional total. Además, producen 600.000 docenas de huevos, existiendo una población aproximada entre 8 a 10 millones de aves de patio, donde no se realizan con frecuencia estudios sobre la calidad e inocuidad del huevo. (Andino \& Rodriguez , 2013)

A nivel nacional existen organizaciones tales como el INTA, que se encargan de brindar charlas educativas a las familias de las zonas rurales del país, con el fin de que se emplee un mejor manejo del ave y sus derivados como es el huevo, para que puedan obtener un producto de calidad libre de contaminantes y con un valor nutricional más alto.

ANAPA en conjunto con la Comisión Nicaragüense del Huevo $(\mathrm{CNH})$, conformada por los productores de huevos de Nicaragua, trabajan para promover los valores nutricionales de este alimento y han trabajado para concientizar a la población sobre los beneficios y las bondades del huevo. La avicultura representa el $30 \%$ del valor agregado del sector pecuario Nicaragüense y aporta el $2.5 \%$ del valor agregado de la economía nacional, son pocas las actividades que en forma específica como la avicultura, ofrecen un aporte de este nivel a la economía de una nación. (Anapa, 2015)

(Juarez, Gutiérrez, Correa, \& Santos, 2010) Realizaron un estudio sobre la calidad del huevo de gallinas criollas criadas en traspatio en Michoacán, México, donde se determinó la calidad de 385 huevos de 11 municipios (35 huevos/municipio), realizando diferentes pruebas de calidad en el que concluyeron que los huevos de gallinas de traspatio, presentan dimensiones reducidas en comparación con el huevo comercial. Sin embargo los indicadores de calidad interna sugieren que el huevo de gallina de traspatio tiene calidad similar al de gallinas de la estirpe comercial.

Por lo antes mencionado, este trabajo busca conocer si los huevos provenientes de gallinas domesticas cumplen con los rangos de calidad, comprobando que sean aptos para el consumo humano y su comercialización, así como el manejo que le dan las familias de la zona al huevo para su consumo. De la misma manera, se pretende detectar la presencia de Salmonella spp en huevos procedentes de gallinas de patio.

\section{Materiales y métodos}

\subsection{Ubicación geográfica del estudio}

El estudio se realizó en la comunidad Piedra Larga Arriba, perteneciente al municipio de Condega, departamento de Estelí, que se encuentra a $12 \mathrm{~km}$ al sur de la cabecera municipal. Se localiza en las coordenadas geográficas Latitud: 13.3 norte, Longitud: 86.36 oeste, Altitud: $768.00 \mathrm{~m} / 2519.69 \mathrm{ft}$, y una elevación de 768 metros/ 2519.69 pies, con precipitaciones promedios de $844 \mathrm{~mm}$. (Condega, 1994) (Ver imagen 1)

\subsection{Población}

La comunidad cuenta con 480 habitantes distribuidos en 100 viviendas, de las cuales se realizó 
una encuesta a 30 casas donde solo en 19 de estas, son dueños de aves de traspatio; obteniendo un total de 141 gallinas ponedoras.

\subsection{Muestra}

La muestra fue de 95 huevos, tomando 5 huevos por cada productor para la realización de las respectivas pruebas.

\subsection{Tipo de estudio}

Se realizó un tipo de estudio descriptivo de corte transversal, que permitió analizar la composición física, química y detección de salmonella spp en huevos de traspatio.

\subsection{Variables de estudio}

Estado físico-químico del huevo: Representa cada una de las etapas o formas, que físicamente se pueden apreciar a través de la medición de ciertas propiedades, que pueden tener un sistema físico o su desarrollo temporal.

\section{Presencia de salmonella en cáscara de huevos:} La salmonella es una enfermedad bacteriana frecuente, que afecta el aparato intestinal; los humanos se infectan mediante alimentos contaminados.

Tipo de manejo aplicado en huevo: Consiste en la acción de manejar o manipular el huevo antes del consumo que realizan los pequeños productores.

\section{Resultados y discusión}

\subsection{Estado físico y químico del huevo Altura de cámara de aire}

Según las mediciones realizadas en cuanto a la altura de la cámara de aire, la figura refleja que del total de huevos estudiados, 22 presentaron una cámara de aire de tres $\mathrm{mm}$ de altura y 19 huevos de dos $\mathrm{mm}$, los cuales entran entre los rangos establecidos por (Pérez, 2009), al no sobrepasar los seis mm, (Salazar Sáenz, 2008) expresa que el huevo fresco carece prácticamente de cámara de aire al hallarse las dos membranas adheridas entre sí (membrana interna y externa).

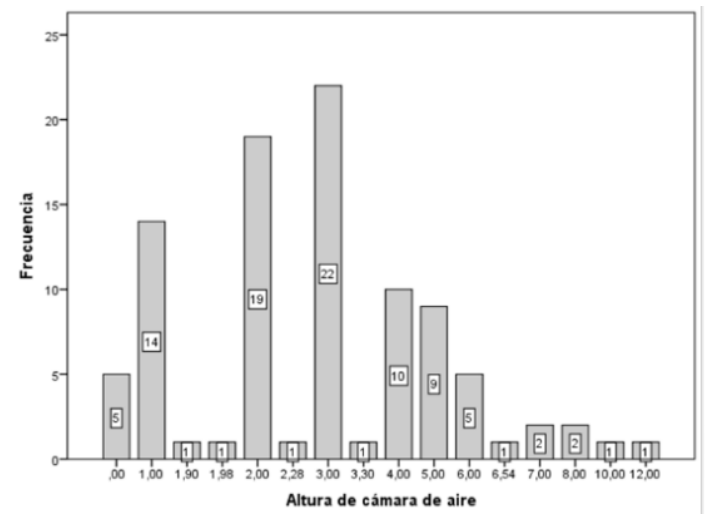

Figura 1. Medición de altura en cámara de aire

(Raigon , Garcia Martinez, \& Esteve, 2019) Realizaron un estudio en el que concluyeron que la cámara de aire de los huevos que proceden de la avicultura intensiva es significativamente mayor (2.54 $\mathrm{mm}$ ) que la de los huevos de procedencia ecológica $(1.79 \mathrm{~mm})$, lo que indica que los huevos procedentes de la avicultura ecológica tienen menos permeabilidad en la cáscara y una mejor formación del huevo, dado que su cámara de aire que presentan en promedio es menor, por lo que mantienen su frescura durante más tiempo.

\subsection{Clasificación del huevo por peso}

Referente a la categoría del huevo, en la gráfica se refleja que de los 95 huevos estudiados, 48 presentaron un peso menor de $53 \mathrm{gr}$ que corresponden a la categoría $S$, seguido por la clasificación $M$, estos se encuentran en las categorías bajas, según (Periago Castón, 2012) los rangos establecidos para medir la calidad del huevo según su peso son $\mathrm{XL}$ : de $73 \mathrm{~g}$ o más, L: de $63 \mathrm{~g}-73 \mathrm{~g}$, M: de $53 \mathrm{~g}$ a $63 \mathrm{~g}$ y S: menos de 53g. (Garcilla, Berrocal, \& Ferrón, 2008) Refieren que el principal componente del huevo que determina su 
tamaño o peso, es el tamaño de la yema cuando es liberada por el ovario, lo cual está muy influido por el peso de la gallina. (Avinews, 2017) Indica que el peso del huevo de gallina está influenciado por la genética, peso corporal y la nutrición.

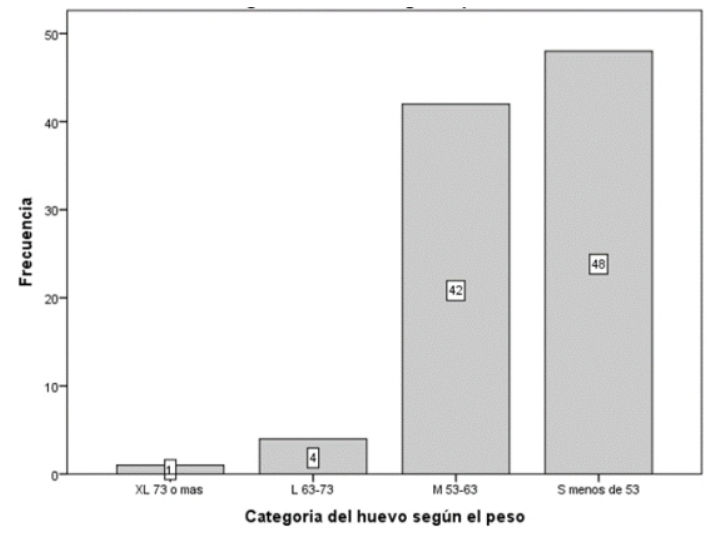

Figura 2. Categoría del huevo según el peso

(Osorio García, 2011) Realizó un estudio en el que le suministró tres tipos de concentrado casero a gallinas de traspatio, donde se pudo determinar que dos de los tratamientos utilizados, mostraron que las gallinas ganaban peso y el peso del huevo aumentaba considerablemente, no así con el tercer tratamiento donde no se observó la misma relación de incremento peso del huevo y peso de la gallina.

\subsection{Posición del huevo en grados}

Esta figura refleja que 26 huevos se encontraron en $0^{\circ}$ grados seguido de 24 huevos con $20^{\circ}$ lo que nos indica que según el rango de grados establecidos para medir el grado de frescura y el tiempo que ha trascurrido luego de la ovoposición es menor de cinco días, siendo huevos frescos en su mayoría. (López A., 2014) Indica que los ángulos en los que se posiciona el huevo de $0^{\circ}$ y $20^{\circ}$ refieren que los huevos tienen de tres a cinco días de almacenamiento.

Con el transcurso del tiempo y en función de las condiciones de almacenamiento se producen en el huevo dos fenómenos que le hacen perder calidad: la salida de parte del agua del huevo a través de los poros de la cascara en forma de vapor (lo que origina el aumento del tamaño de la cámara de aire y por ende la disminución del peso del huevo), y la eliminación de anhídrido carbónico (Pérez, 2009).

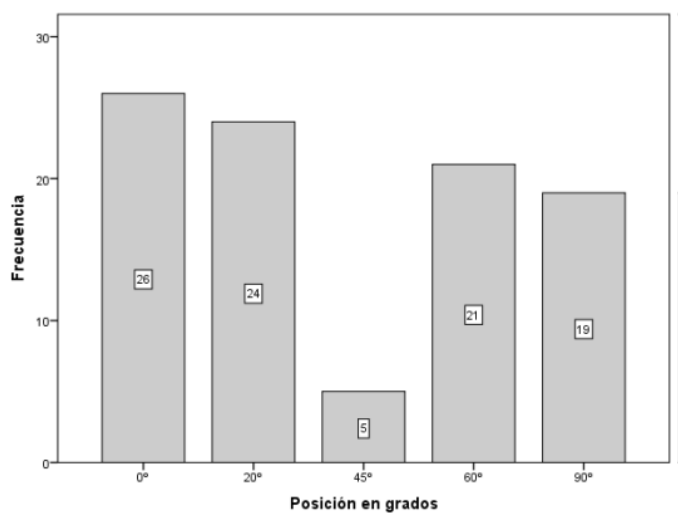

Figura 3. Posición en grados

\section{4 Índice de la clara}

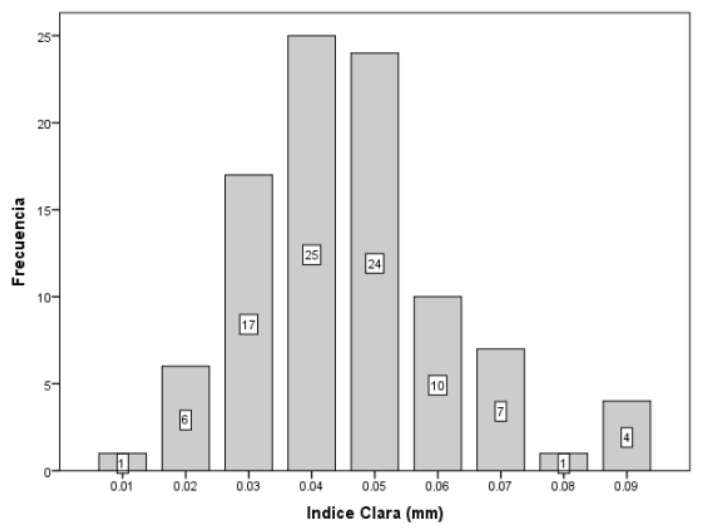

Figura 4. Índice de la clara

En la figura 4 se muestra que 25 huevos presentaron un índice de clara de $0.04 \mathrm{~mm}$, seguido de 24 huevos con $0.05 \mathrm{~mm}$. Según (López, Pinillos, \& Pérez, 1997) refieren que los rangos indicados son de $(0,07 \mathrm{~mm}-0,11 \mathrm{~mm})$ así mismo señalan que este indicador es uno de los más importantes sobre la calidad interna y que se afecta más rápidamente que el índice de yema, sobre todo cuando las condiciones del almacenamiento no son las ideales. Referente a lo dicho se indica que los huevos muestreados están fuera del rango establecido, en su mayoría se encuentran bajos. 
(Casas Rodríguez, Guerra Casas, Ceró Rizo, \& Uña Izquierdo, 2016), realizaron un estudio sobre empleo de los diámetros del huevo para el cálculo del volumen y superficie y su correlación con otros caracteres externos e internos en tres propósitos de gallinas reproductoras en el cual no encontraron diferencias significativas entre los propósitos para el índice de clara ya que estaban en los rangos establecidos, esto no coinciden con el resultado de este estudio puesto que los resultados obtenidos se encuentran bajos según los rangos.

\subsection{Color de yema en huevos}

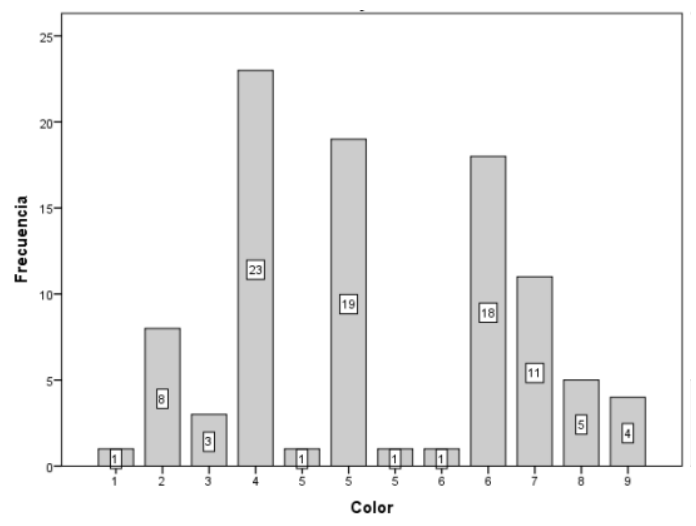

Figura 5. Color de yema

En la figura 5 se observa que 23 huevos se encontraron en la escala cuatro y seguido de 21 huevos ubicados en la escala cinco, lo que indica que la coloración de los huevos estudiados es baja. El color de la yema se evaluó a través del valor de Roche asignado por la escala colorimétrica mediante comparación. (Nieves Viñas, 2015) refiere que la escala de Roche va de 1 al 15, sugiriendo que 11 y 12 son los valores óptimos en la pigmentación del huevo, así mismo refiere que los huevos no alcanzan las escalas optimas de pigmentación ya que el contenido de pigmentos en las materias primas no es suficiente para conseguir una pigmentación adecuada de la yema.

(H. Araya, R. Murillo, G. Vargas, \& M. Delgado, 1977) Realizaron un estudio sobre la composición y uso de tres cultivares de achiote (Bixa Orellana), como fuente de pigmentación de la yema de huevo en el que concluyeron que es necesario incluir una cantidad de $203 \mathrm{gr}$ de pigmento provenientes de achiote por tonelada de alimento, con el fin de obtener una coloración entre 9 y 10 de la escala Roche.

\section{$3.6 \mathrm{pH}$ en clara de los huevos}

La figura 6 nos muestra que los resultados obtenidos en el análisis de $\mathrm{pH}$ de clara nos indican que 80 huevos en su mayoría presentaban un $\mathrm{pH}$ de nueve, esto nos refiere que los huevos estudiados sobrepasaron los rangos establecidos de frescura. (Nieves Viñas, 2015) Menciona que los procesos de envejecimiento que se producen en el huevo y que se inician tras la puesta, dan lugar a la liberación de anhídrido carbónico desde el interior del huevo a través de la cáscara, con el consiguiente aumento del $\mathrm{pH}$.

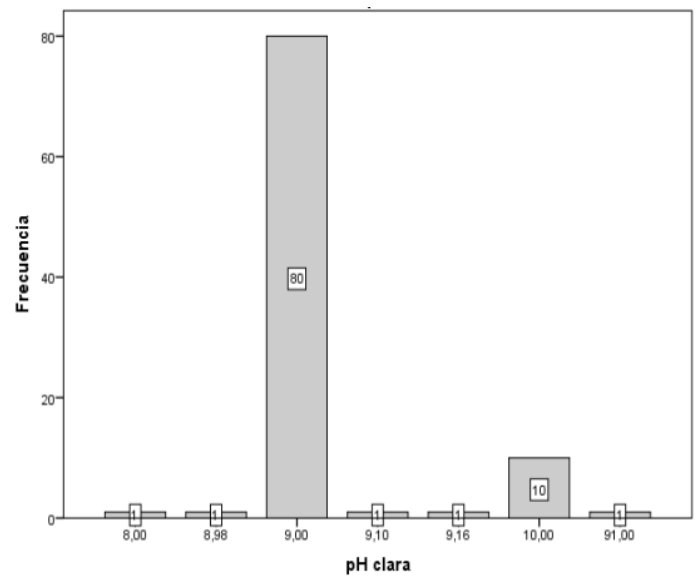

Figura 6. Medición de $\mathrm{pH}$ clara

(Milleni, Folegatti, Sirri, \& Meluzzi, 2010) Observaron que los huevos de gallinas en jaulas convencionales de Italia, presentaban un mayor incremento en el $\mathrm{pH}$ de la clara debido a la elevada concentración de amoniaco en el ambiente de este tipo de producción. Esto no concuerda con los resultados obtenidos en esta investigación sin embargo puede verse la diferencia en el ambiente, producción y manejo de las aves en distintas partes del mundo. 


\section{$3.7 \mathrm{pH}$ de yema en huevos}

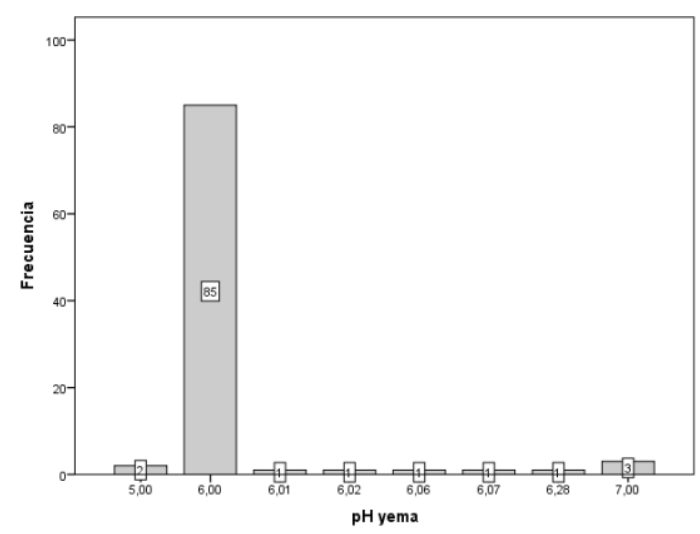

Figura 7. Medición de $\mathrm{pH}$ yema

La figura indica que de las 95 muestras estudiadas, 85 huevos presentaron un $\mathrm{pH}$ en la yema, de seis. No se observó una variación significativa con el resto de resultados de esta prueba, lo que refiere a que se encontraban dentro de los rangos establecidos para $\mathrm{pH}$ de yema. La yema tiene un $\mathrm{pH}$ cercano a seis que se eleva durante el almacenamiento del huevo $y$ alcanza valores entre 6.4 y 6.9 (Pacual \& Calderón, 2000). El incremento no es tan pronunciado en vista que la yema no es tan susceptible a cambios, pudiendo deberse a que esta presenta una composición lipoproteíca que minimiza su deterioro (Arias \& Fernández, 1998).

(Rosero, 2015) Determinó que el pH en yema incrementa conforme transcurre el tiempo con menor pronunciamiento en esta, al igual que la variación de $\mathrm{pH}$ de yema en el tiempo en función de la temperatura, observándose que dicho incremento no presenta tendencia uniforme bajo diferentes condiciones de almacenamiento.

\section{8 Índice de yema en huevos}

De los resultados obtenidos que muestra la figura 9 solamente ocho huevos de los 95 estudiados, se encuentran en los rangos establecidos para el índice de yema, en su mayoría bajos; Según (Martín Gairal, 2015) el índice de yema normal aproximadamente es de $0.40-0.42$, cuando se supera esta cifra significa que la yema ha descendido debido a que la membrana vitelina ha adquirido mayor elasticidad y ha perdido más agua o humedad. El índice de yema es un parámetro que informa sobre la forma ideal de la yema y su relación con la frescura y calidad del huevo. Cuanto mayor sea el valor de este índice, mayor es la frescura del huevo, ya que la yema se presenta más compacta. (Periago Castón, M J, 2012)

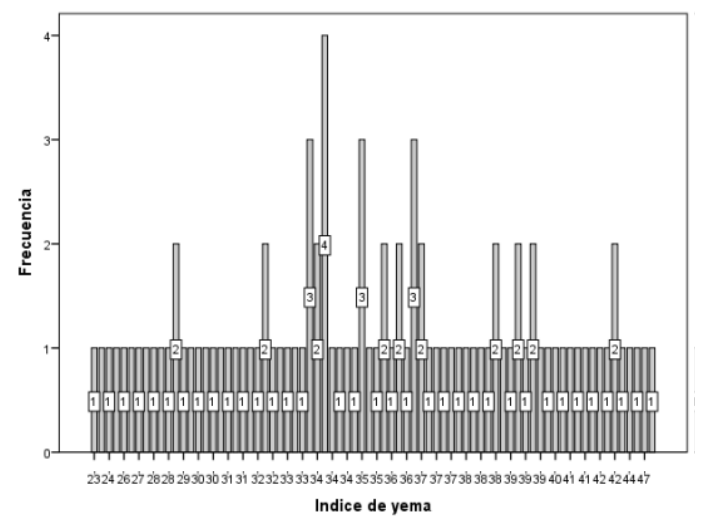

Figura 8. Índice de Yema

(Estrada, Galeano, \& Her, 2010) Realizaron un estudio sobre el efecto de la temperatura y el volteo durante el almacenamiento sobre la calidad del huevo comercial, en el que concluyeron que a pesar del trayecto del tiempo, el índice de yema no presenta cambios significativos al ser conservados bajo un ambiente de refrigeración, a diferencia de conservarlos a temperatura ambiente con el tiempo el índice de yema decreció.

\subsection{Análisis de grado de frescura (unidades Hauhg)}

En esta figura se muestra que hay una variación de frescura entre los 95 huevos, donde la mayoría siendo en este caso 39 nos refieren que se encuentran en el rango inaceptable, (Nieves Viñas, 2015) indica que comparando con los valores de referencias de las unidades Hauhg, podemos determinar la calidad del huevo en función de la clara.

(Juarez, Gutiérrez, Correa, \& Santos, 2010) Realizaron un estudio de calidad de huevos de gallinas 
criollas criadas en traspatio, donde refiere que el huevo muestreado almacenado durante un periodos de tres a siete días, la calidad interna del huevo varia de mínima a buena, los promedios obtenidos en las unidades Haugh fueron de 73.4 .

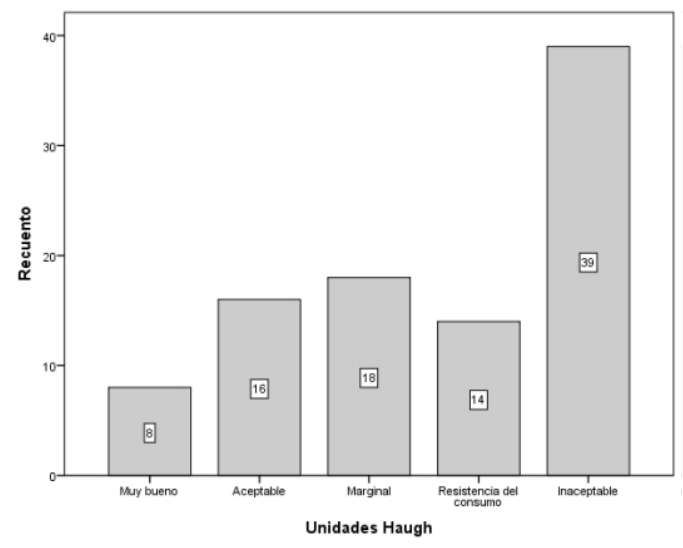

Figura 9. Unidades Haugh

\subsection{Determinación de presencia de salmonella en cáscara de los huevos estudiados}

Mediante la realización de cultivos en agar Salmonella-Shigela se determinó la presencia de Salmonella en una de las muestras que correspondía a la casa número cuatro como se muestra en la tabla.

(Salazar, Villamarín, Gómez M, Camacho, \& Rojas, 2015) Realizó un estudio sobre la detección e identificación de Salmonella spp en huevos para consumo humano, provenientes de diferentes localidades de Bogotá, Colombia en el que se realizaron dos muestreos donde se concluyó que de las 96 muestras obtenidas en las cuatro localidades, se detectó un $9,4 \%$ de muestras positivas para Salmonella spp.; de éstas, el $55 \%$ provenían del contenido interno y $44 \%$ de cáscara.

\subsection{Tipo de alimentación que los productores suministran a las aves}

En la comunidad encuestada, las familias proporcionan a sus aves básicamente maíz como alimento primario, como se muestra en la figura, ya que se dedican a la producción de este grano y les resulta más económico dárselo a los animales que optar por otras fuentes de alimentación. El alimento comercial casi no se proporciona a los animales porque consideran que tiene un elevado costo, ellos carecen de suficientes recursos económicos y no se expenden en sus comunidades de modo que tendrían que comprarlo en la cabecera departamental y eso implica incurrir en gastos de transporte adicionales.

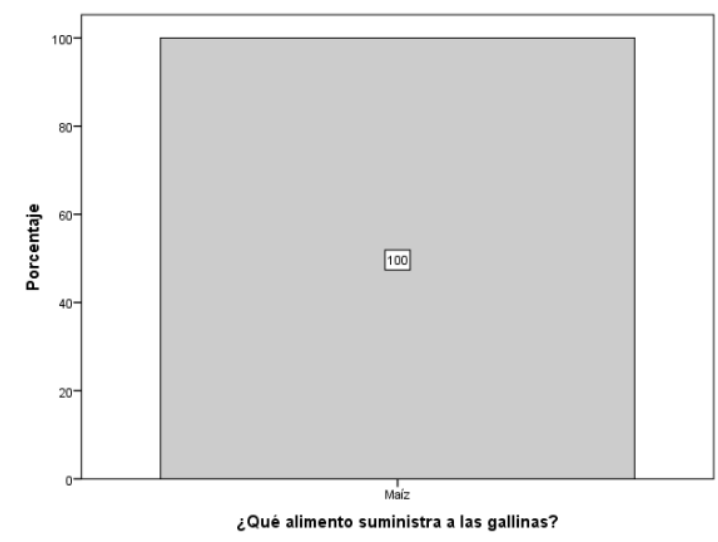

Figura 10. Alimentos suministrados a las gallinas

En un estudio realizado en los municipios de Ignacio de la Llave y Teocelo en Veracruz-México se menciona que las aves de traspatio se alimentan principalmente a base del grano del maíz y secundariamente con desperdicios de cocina y hiervas que ingieren en el área en que se confinan (Molina Martínez, 2013)

\subsection{Limpieza que se le realiza al huevo}

La figura 11 muestra que un $57.89 \%$ de los productores encuestados no le realizan ningún tipo de limpieza a los huevos desde que son tomados del nido hasta su consumo. El $26.32 \%$ señalaron realizar un enjuague a los huevos atribuyendo que los huevos por su trayectoria fisiológica en la gallina y al ser depositados, traen consigo suciedad en la cáscara.

De acuerdo a (USDA, 2013) el proceso de lavado elimina el recubrimiento natural de los huevos recién puestos, que ayuda a evitar que las bacterias penetren 
en la cáscara. El lavado, podría aumentar el riesgo de contaminación cruzada, especialmente si la cáscara se agrieta. Así mismo (Mercree, 2019) Advierte que si se lava un huevo antes de cocinarlo, su cáscara porosa y el agua puede introducir las bacterias al huevo, especialmente si utiliza agua fría o agua corriente.

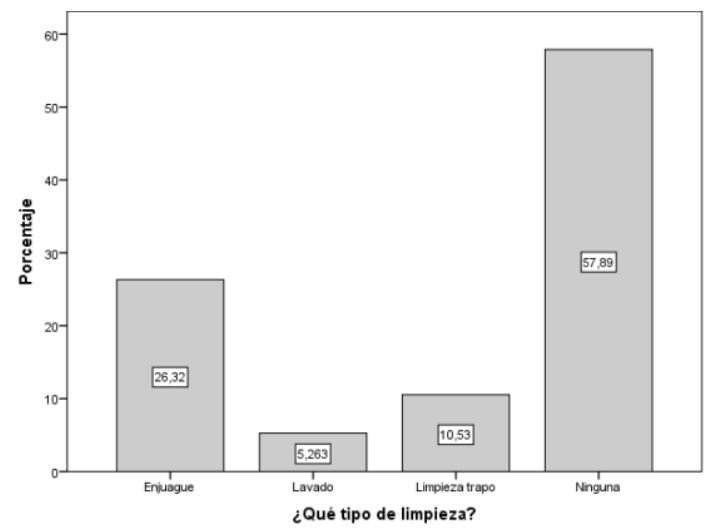

Figura 11. Limpieza que se realiza al huevo

\subsection{Sitio de ovoposición}

Los sitios donde las aves ovopositan son muy variados como lo muestra figura 12 , los sitios de las aves de la comunidad para la ovoposición son los nidos con un $52.63 \%$ de preferencia debido a que en su mayoría los productores tiene a las aves confinadas en gallineros, en cuanto al $21,05 \%$ el sitio preferido es el suelo esto es debido a que las aves que se encuentran en libertad no cuentan con un área específica para la ovoposición.

Los sitios donde las aves ovopositan son muy variados como lo muestra figura 12 , los sitios de las aves de la comunidad para la ovoposición son los nidos con un $52.63 \%$ de preferencia debido a que en su mayoría los productores tiene a las aves confinadas en gallineros, en cuanto al $21,05 \%$ el sitio preferido es el suelo esto es debido a que las aves que se encuentran en libertad no cuentan con un área específica para la ovoposición.

(Villanueva, y otros, 2015). Indican que los nidales - nidos proporcionan sitios en penumbra $y$ semiocultos, para que las gallinas realicen la postura con comodidad y eviten picar los huevos. Son de suma importancia a la hora de recolectar huevos en condiciones adecuadas y prevenir pérdidas (huevos que se quiebran o contaminan).

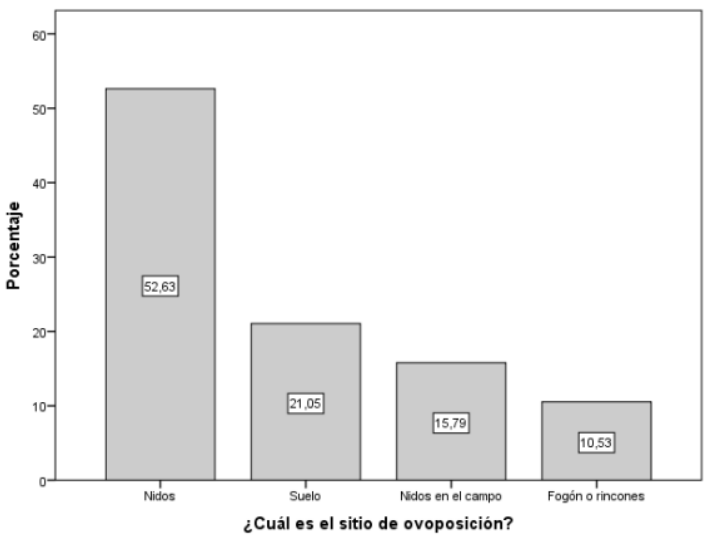

Figura 12. Sitio de ovoposición

\subsection{Almacenamiento de los huevos}

La figura refleja que un $76.92 \%$ en su mayoría los productores utilizan panas para almacenar los huevos que producen sus gallinas y un $23.08 \%$ los almacenan en canastas, esto es debido a que piensan que es un producto que no requiere condiciones muy específicas para su manejo y conservación.

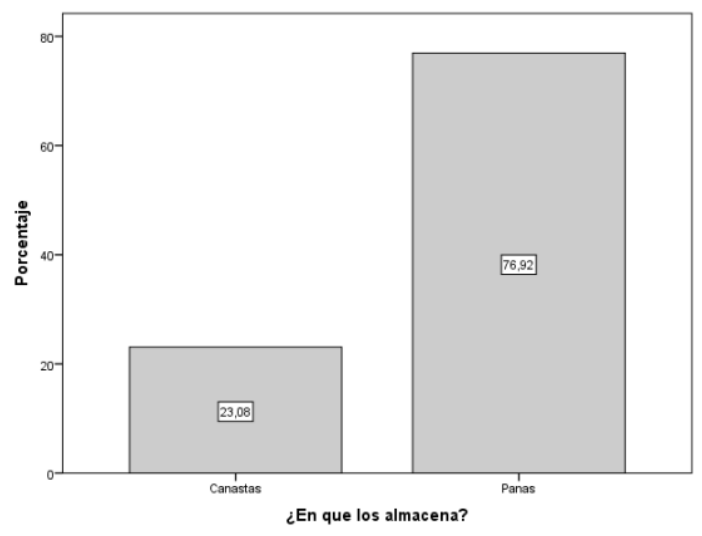

Figura 13. Almacenamiento de los huevos

En relación a lo antes mencionado los productores realizan un buen almacenamiento de los huevos ya que (Télles Flores, 2011) sugiere que los huevos se 
deben almacenar en un recipiente seguro (caja, pana $\mathrm{u}$ otro) con el extremo (polo) más puntiagudo hacia abajo para que el espacio de aire que hay dentro del huevo quede en la parte superior y conserve su calidad.

\subsection{Tipo de limpieza que le realiza a los gallineros}

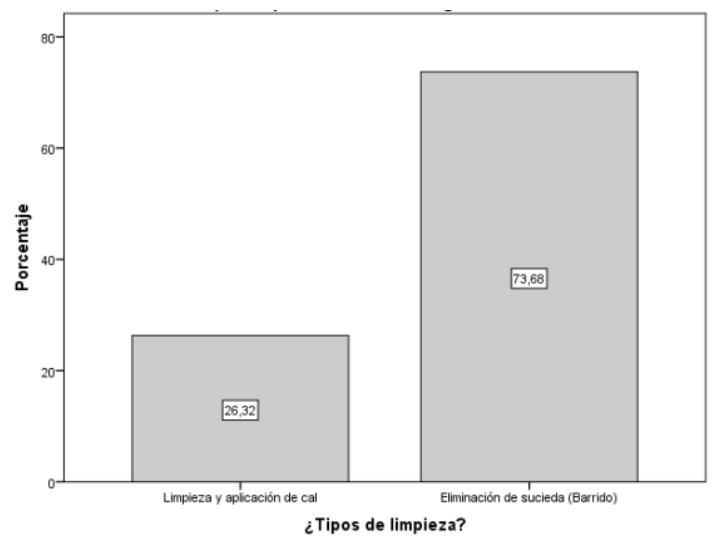

Figura 14. Tipos de limpieza que se realiza en los gallineros

Esta figura muestra que según los encuestados solo un $73.68 \%$ realizan lo que es la eliminación de suciedad presente en los gallineros, debido a que las áreas donde están confinadas las aves son gallineros rústicos sin ningún tipo de tecnificación; respecto al $26,32 \%$ utilizan únicamente cal como método de desinfección del área.

En la comunidad carecen de la implementación de programas higiénicos en cuanto al área de los gallineros. (Sanchéz, C, 2013) Sugiere que basta con limpiar el gallinero una vez por semana o cada dos semanas, procurando hacerlo a conciencia y llegando a todas las esquinas, con el fin de evitar que aparezcan parásitos $\mathrm{u}$ otros visitantes inesperados como ácaros. El proceso general de limpieza es sencillo en sí, y solamente constaría de dos pasos; quitar las virutas o suciedad viejas del gallinero.

\section{Conclusiones}

El análisis realizado mediante las diferentes pruebas en los huevos de gallina de traspatio de la comunidad Piedra Larga, mostró una variabilidad en cada prueba, estando entre los rangos normales la cámara de aire con $3 \mathrm{~mm}$ en su mayoría, gravedad especifica mediante la posición en grados del huevo con $0^{\circ}$ y $20^{\circ}, \mathrm{pH}$ en yema de 6 e índice de yema con $0.34 \mathrm{~mm}$, lo que indica que poseen cierta calidad de frescura.

En cuanto a los resultados para el grado de frescura mostraron valores igual a 50 unidades Haugh refiriendo que son inaceptables según los rangos establecidos, así mismo se mostró un incremento en el pH de la clara, esto debido a los días de almacenamiento; referente al peso en su mayoría son menor a 53gr siendo estos según la clasificación de menor tamaño, mediante estas pruebas se determinó que los huevos son de baja calidad.

Respecto a la detección de salmonella en cáscara mediante la realización de cultivos específicos de agar, solamente en una muestra se detectó la presencia de Salmonella spp, siendo este un valor mínimo a pesar de las condiciones ambientales y de manejo que realizan en la zona.

El tipo de manejo que se realiza a los huevos es convencional, debido a que la mayoría de los productores se limitan a recoger los huevos $y$ almacenarlos hasta el día de su consumo. La avicultura de esta zona está enfocada principalmente en la producción de huevos para el autoconsumo y la venta de estos.

\section{Referencias}

Anapa. (2015). Anapa. Obtenido de Asociasion nacional de avicultores y productores de alimentos: http://www.anapa.org.ni/

Andino, M., \& Rodriguez , Y. (Octubre-noviembre de 2013). unanleon.edu.ni. Obtenido de http://riul.unanleon.edu.ni:8080/jspui/bitstream/123456 789/3450/1/225912.pdf

Arias, J. L., \& Fernández, M. S. (12 de 1998). ¿Que se entiente por un huevo fresco? TecnoVet, 4(3). Obtenido 
Moreno Castellón, M. de J., Velasquez Raudez, E. J., \& Moreno Rivera, M. A. (2021). Análisis físico, químico y detección de salmonella spp en huevos provenientes de gallus gallus domesticus, comunidad Piedra Larga, Condega 2018-2019. Teknos Revista Cientifica, 21(1), 16-

http://web.uchile.cl/vignette/tecnovet/CDA/tecnovet_ar ticulo/0,1409,SCID\%253D9610\%2526ISID\%253D458, 00.html

Avinews. (abril de 2017). Mejorando el tamaño del huevo en las ponedoras comerciales. aviNews. Obtenido de https://avicultura-

info.cdn.ampproject.org/v/s/avicultura.info/mejorandoel-tamano-del-huevo-en-las-ponedoras-

comerciales/amp/?usqp=mq331AQDoAEC\&amp_js_v $=0.1 \#$ aoh $=15583975601843 \&$ \&amp_ct $=155839765863$

$2 \&$ csi $=1 \&$ referrer=https $\% 3 \mathrm{~A} \% 2 \mathrm{~F} \% 2 \mathrm{Fwww}$.google.com \&amp_tf=De\%20\%

Ballina G, A. (Enero de 2008). Fao. Obtenido de http://www.fao.org/3/a-as541s.pdf

Calero, C. (Abril de 2018). Conocer la Agricultura y Ganaderia. Obtenido de Conocer la Agricultura y Ganaderia:

http://www.conocerlaagricultura.com/2018/04/de-lasyemas-de-los-huevos-su-color-y.html?m=1

Cano Villalba, M. A. (19 de Enero de 2016). Catelló. Obtenido de https://www.naturalcastello.com/propiedades-valornutricional-huevo/

Casas Rodríguez, S., Guerra Casas, L., Ceró Rizo, Á., \& Uña Izquierdo, F. (2016). Empleo de los diámetros del huevo para el cálculo del volumen y superficie y su correlación con otros caracteres externos e internos en tres propósitos de gallinas reproductoras en tres propósitos de gallinas reproductoras, Tesis. Facultad de Ciencias Agropecuarias, Universidad de Camagüey Ignacio Agramonte Loynaz, Cuba. Obtenido de http://scielo.sld.cu/pdf/rpa/v28n2-3/rpa05216.pdf

Chabarría, M. (13 de Agosto de 2014). El tiempo de almacenamiento de huevo más seguro. Consumer. Obtenido de http://www.consumer.es/seguridadalimentaria/sociedad-yconsumo/2014/08/13/220420.php

Cienfuegos, C. (2008). El huevo como aliado enla nutrición y alimentación. Revista cubana, 18(2), 15. Obtenido de http://www.revicubalimentanut.sld.cu/Vol_18_2/Resum enes $\% 20$ Seminario $\% 20$ Huevo.pdf

Clinic, P. d. (07 de Septiembre de 2018). Mayo CLINIC. Obtenido de https://www.mayoclinic.org/eses/diseases-conditions/salmonella/symptomscauses/syc-20355329

Condega, A. d. (1994). Mejoramiento del sistema de abastecimiento de agua y saneamiento en la comunidad de Piedra Larga arriba. Proyecto, Esteli. Obtenido de http://doczz.es/doc/6157992/perfil-piedralarga-arriba
Cozano Rubio, L. F. (Noviembre de 2003). Evaluacion sanitaria (fisico, quimico, bacteriologico) del huevo de gallina de traspatio, en expendios del mercado de la terminal, zona cuatro de la ciudad de Guatemala. Obtenido de www.repositorio.usac.edu.gt

EcuRed. (16 de octubre de 2012). Obtenido de conocimiento con todos y para todos: https://www.ecured.cu/Huevo

Estrada, M. M., Galeano, L. F., \& Her, M. (13 de Abril de 2010). Efecto de la temperatua y del volteo durante el almacenamiento sobre la calidad del huevo comercial. Colombiana de ciencias pecuarias, 23(2), 23:183-190. Obtenido de http://aprendeenlinea.uda.edu.co/revistas/intex.php/rc cp/article/view/324561/20781779

Estrada, M., Galeano, L., Herrera, M., \& Restrepo, L. (13 de 4 de 2010). Revista colombiana de ciencias pecuarias, 8. Obtenido de https://aprendeenlinea.udea.edu.co/revistas/index.php /rccp/article/view/324561/20781780

Etxebarria, P. (s.f.). Laboratorio del Huevo. Obtenido de https://sites.google.com/site/laboratoriodelhuevo/quimi huevo/tipos-de-huevos

FAO. (2013). Organización de las Naciones Unidas para la Alimentación y la Agricultura. Obtenido de Organización de las Naciones Unidas para la Alimentación y la Agricultura: http://www.fao.org/3/ai3351s.pdf

García Trujillo, , R., \& Berrocal, J. (s.f.). Produccion Ecologica de Gallinas ponedoras. Obtenido de https://www.juntadeandalucia.es/opencms/opencms/s ystem/bodies/contenidos/publicaciones/pubcap/2009/ pubcap_2931/Produccixn_EcolxgicaGallinasPonedora s_baja.pdf

Garcia Zubia, J. (s.f.). WEblab. Obtenido de Composicion fisica del huevo: https://weblab.deusto.es/olarex/cd/UD/Incubator_ES_f inal/caractersticas_fsicas.html

Garcilla, R., Berrocal, J., \& Ferrón, G. (2008). Fundesyram. Obtenido de Fundesyram: https://www.juntadeandalucia.es/opencms/opencms/s ystem/bodies/contenidos/publicaciones/pubcap/2009/ pubcap_2931/Produccixn_EcolxgicaGallinasPonedora s_baja.pdf

Gonzalez Ortis, F., Magaña Pérez, A., Ocampo Fletes, I., Paredes Sánchez, J., \& Peñaloza, P. d. (junio de 2013). Obtenido de https://www.ciad.mx/archivos/revistaeletronica/RES44/Floriberto_Gonzalez.pdf

H. Araya, H., R. Murillo, M., G. Vargas , E., \& M. Delgado, J. (1977). composicion y empleo del achiote (Bixa Orellana) en raciones para gallinas ponedoras, parala 
Moreno Castellón, M. de J., Velasquez Raudez, E. J., \& Moreno Rivera, M. A. (2021). Análisis físico, químico y detección de salmonella spp en huevos provenientes de gallus gallus domesticus, comunidad Piedra Larga, Condega 2018-2019. Teknos Revista Cientifica, 21(1), 16-

pigmentacion de la yema del huevo. Obtenido de http://www.mag.go.cr/rev_agr/v01n02_143.pdf

Huevo, I. d. (06 de 15 de 2013). Instituto del huevo. Recuperado el 30 de Noviembre de 2018, de http://www.institutohuevo.com/estructura_huevo/

Inta. (6 de Febreo de 2014). Manejo de gallinas de patio. Obtenido de http://wwww.inta.gob.ni

Juarez, A., Gutiérrez, E., Correa, J., \& Santos, R. (12 de Enero-Abril de 2010). CALIDAD DEL HUEVO DE GALLINAS CRIOLLAS CRIADAS EN TRASPATIO EN MICHOACAN, MEXICO. Tropical and Subtropical Agroecosystems.

Obtenido

de

http://www.redalyc.org/pdf/939/93913074011.pdf

Licata , M. (2018). Zonadiet. Obtenido de Zonadiet: https://www.zonadiet.com/comida/huevopropiedades.htm

López, A. (25 de 01 de 2014). Slideshare. Obtenido de https://es.slideshare.net/ALEJONIO01/el-huevo-y-sussubproductosfin

López, A., Pinillos, M., \& Pérez, E. (1997). Manual de teoría, cría y explotación de las aves. Cuba.

M.A.R.M. (2013). Ministerio de Agricultura, Pesca y Alimentación . Obtenido de http://www.alimentacion.es/es/conoce_lo_que_comes/ bloc/huevo/el-huevo-por-dentro/estructura/

Martín Gairal, N. (26 de 10 de 2015). Veterinaria digital. Obtenido de https://www.veterinariadigital.com/articulos/calidadinterna-del-huevo/

Mercade, A. (22 de Septiembre de 2010). Transformando el Infierno. Obtenido de https://transformandoelinfierno.com/2010/09/22/elhuevo-formacion-estructura-y-composicion/

Mercree, A. L. (05 de Marzo de 2019). El sol de Tlaxcala. Obtenido de El sol de Tlaxcala: https://www.elsoldetlaxcala.com.mx/doble-via/por-queno-se-deben-lavar-los-huevos-antes-de-cocinarlos3146274.html

Milleni, G., Folegatti, E., Sirri, F., \& Meluzzi, A. (04 de 2010). Rasgos de calidad de huevo de gallinas ponedoras criadas en sistemas orgánicos y convencionales. Obtenido de https://www.researchgate.net/publication/44121950_E gg_quality_traits_of_laying_hens_reared_in_organic_ and_conventional_systems

Molina Martínez, P. (2013). Comparación de dos sistemas de producción y de manejo sanitario de las aves criollas de traspatio en el municipiode Ignacio de la Llave y Teocelo (Tesis). Tesis, Veracruz. Obtenido de https://www.uv.mx/veracruz/uvca366-agronegocios-
sustentables/files/2013/12/Molina2013-Aves-detraspatio-Tesis.pdf

Nieves Viñas, A. (2015). Control y manejo de huevos y pollos recien nacidos en la explotacion avícola. Obtenido de https://books.google.com.ni/books?id=12BWDwAAQB AJ\&pg=PA119\&lpg=PA119\&dq=posicion $\% 20$ en $\% 20 \mathrm{gr}$ ados $\% 20$ de $\% 20$ los $\% 20$ huevos \&source=bl\&ots=YyAP HwP75f\&sig=ACfU3U2LrPdXQ0ipDVvoD5kB7sv6toLE 2Q\&hl=es-419\&sa=X\&ved=2ahUKEwi4bzShbDiAhVQx1kKHZbHDI4Q6AEwD3oECAgQAQ\# $\mathrm{v}=$ onepage

Osorio García, U. E. (2011). Evaluación productiva en gallinas de patio, tesis. Managua, Nicaragua, Nicaragua : UNA. Obtenido de http://repositorio.una.edu.ni/1432/1/tnl02o83.pdf

Pacual, M. d., \& Calderón, V. (2000). Microbiología alimentaria (Vol. segunda edicion). (S. Díaz, \& J. Bravo, Edits.)

Pastor, A. M. (noviembre de 2009). Manipulacion e higiene de alimentos. Obtenido de Manipulaicon e higiene de alimentos:

https://alimentosmanipulacion.blogspot.com/2009/11/e I-huevo.html

Pérez, D. R. (2009). El gran libro del huevo (Vol. Primera edicion ). Madrid, España: EVERGRAFICAS, S.L.

Periago Castón, M J. (2012). Higiene, inspeccion y control de huevos de consumo. Universidad de Murcia. Obtenido de https://www.um.es/documents/4874468/10812050/pro tocolos-control-de-calidad-huevos.pdf/c860b16b-6c2f481a-9d52-542a2296d005

Periago Castón, M. J. (2012). Higiene, inspeccion y control de huevos de consumo. Universidad de Murcia. Obtenido de https://www.um.es/documents/4874468/10812050/pro tocolos-control-de-calidad-huevos.pdf/c860b16b-6c2f481a-9d52-542a2296d005

Raigon, Garcia Martinez, \& Esteve. (2019). VALORAClóN DE LA CALIDAD DEL HUEVO DE GRANJA ECOLóGICA E INTENSIVA. Obtenido de https://www.researchgate.net/publication/237351297 VALORACION_DE_LA_CALIDAD_DEL_HUEVO_DE _GRANJA_ECOLOGICA_E_INTENSIVA

Ramírez Rueda, R. Y., Rincón Acero, D. P., \& Vargas Medina, J. C. (2011). Transmisión de Salmonella enterica a través de huevos de gallina y su importancia en salud pública. Salud UIS, 43(2). Obtenido de https://revistas.uis.edu.co/index.php/revistasaluduis/ar ticle/view/2402 
Moreno Castellón, M. de J., Velasquez Raudez, E. J., \& Moreno Rivera, M. A. (2021). Análisis físico, químico y detección de salmonella spp en huevos provenientes de gallus gallus domesticus, comunidad Piedra Larga, Condega 2018-2019. Teknos Revista Cientifica, 21(1), 16-

Rosero, R. E. (2015). Evaluación fisica, quimica y microbiologica de huevos comerciales de gallinas. experimental, UNIVERSIDAD CENTRAL DEL ECUADOR , Quito. Obtenido de http://www.dspace.uce.edu.ec/bitstream/25000/6434/1 /T-UCE-0008-095.pdf

Salazar Sáenz, Y. G. (Julio de 2008). USAC. Recuperado el 2019, de USAC: http://www.repositorio.usac.edu.gt/7154/

Salazar, R., Villamarín, A., Gómez M, F. M., Camacho, A., \& Rojas, K. L. (2015). Detección e identidicación de Salmonella spp. en huevos para consuno humano, provenientes de diferentes localidades de Bogotá Colombia 2015. Bogotá. Obtenido de http://www.scielo.org.co/pdf/inf/v21n3/0123-9392-inf21-03-00154.pdf

Salvador, E. (18 de mayo de 2018). Actualidad Avipecuaria. Recuperado el 30 de noviembre de 2018, de http://www.actualidadavipecuaria.com/articulos/calida d-interna-del-huevo-causas-y-manejo-de-laincidencia-de-manchas-de-sangre-carne-en-yemaclara-de-huevo-de-gallinas-de-postura-comercial.html

Sanchéz, C, T. (2013). Planetahuerto. Obtenido de Planetahuerto:

https://www.planetahuerto.es/guias/guia-sobre-la-criade-gallinas

Sastre Gallego , A., Ortega Anta, R. M., \& Tortuero Cosialls, F. (2003). El libro del huevo. Madrid: Instituto de Estudios del Huevo. Obtenido de http://institutohuevo.com/wp-

content/uploads/2017/07/EL-LIBRO-DEL-HUEVO.pdf

SECO, U. C. (2017). guia de protocolo de tesis . Guia, Esteli , Esteli.

Soler, M. D., Garces , C., \& Barragán, I. J. (26 de 02 de 2015). Portal Veterinario. Obtenido de Portal Veterinario:

https://www.portalveterinaria.com/avicultura/articulos/8 713/la-alimentacion-de-la-ponedora-y-la-calidad-delhuevo.html

Soler Fonseca , D. M. (2010). Importancia de los sistemas avicolas campesinos (pollo de engorde y gallina ponedora). Boyaca, Colombia. Obtenido de https://repository.javeriana.edu.co/bitstream/handle/10 554/852/eam70.pdf?sequence=1\&isAllowed=y

Télles Flores, J. A. (2011). Manual de gallinas de patio. Manual Tecnico, UNA, Managua, Managua. Obtenido de http://repositorio.una.edu.ni/2421/1/nl70t275m.pdf

Téllez Flores, J. A. (Octubre de 2011). Repositorio UNA. Obtenido de Repositorio UNA: http://repositorio.una.edu.ni/2421/1/nl70t275m.pdf

Trijillo Garcia, R., Berrocal, J., Moreno, L., \& Ferrón, G. (2009). Producción ecológica de gallinas ponedoras. Andalucía. Obtenido de https://www.juntadeandalucia.es/export/drupaljda/GAL LINAS\%20PONEDORAS_CUBIERTA\%20E\%20INTE RIOR.pdf

Troncoso, M. C. (2005). Deteccion de Salmonella spp en huevos de gallina comercializados en ferias de la ciudad de Valdivia. Chile. Obtenido de http://cybertesis.uach.cl/tesis/uach/2005/fvc629d/doc/f vc629d.pdf

USDA, D. d. (01 de Julio de 2013). USDA. Obtenido de USDA:

https://www.fsis.usda.gov/wps/portal/fsis/topics/foodsafety-education/get-answers/food-safety-factsheets/safe-food-handling/washing-food-does-itpromote-food-safety/washing-food

Valle, U, A. L., \& Rodriguez, M, J. S. (2013). CARACTERIZACIÓN DEL SISTEMA DE PRODUCCIÓN DE AVES DE PATIO-Tesis. Matagalpa, Nicaragua . Obtenido de http://repositorio.unan.edu.ni/7167/1/6598.pdf

Villanueva, C., Oliva, A., Torres, A., Rosales, M., Moscoso, C., \& Gonzales, E. (2015). Siatma. Obtenido de http://www.siatma.org/sitios/biblioteca/uploads/00015GRAF-SLM.pdf

Villanueva, C., Olivas, A., Torres, Á., Rosales, M., Moscoso, C., \& González, E. (2015). Centro Agronómico Tropical de investigacion (CATIE). Obtenido de Centro Agronómico Tropical de investigacion (CATIE): http://repositorio.bibliotecaorton.catie.ac.cr/bitstream $/ \mathrm{h}$ andle/11554/8001/Manual_de_producion_manejo_ave s_de_patio.pdf

Wikiwand. (s.f.). Obtenido de http://www.wikiwand.com/es/Unidad_Haugh 